\title{
Structure of Financial Savings during Indian Economic Reforms ${ }^{*}$
}

Raghbendra Jha

Australian National University, Canberra, Australia and

Ibotombi S. Longjam,

IGIDR, Mumbai, India

\begin{abstract}
In an economy undergoing structural reforms the composition of savings goes through considerable change. It is important to understand such changes both for increasing the volume of aggregate savings (to garner resources for higher economic growth) as well as affecting their composition (towards more productive instruments) through an understanding of inter-asset substitutability. We conduct nonparametric tests to examine whether data on financial savings in India can be rationalized in terms of a utility function of a representative economic agent. The parametric test has the disadvantage that in some cases it is not possible to distinguish between rejections of the functional form from a rejection of weak separability. We establish that data on financial savings in India are consistent with the existence of a utility function for a representative individual with a sub-preference where contractual savings (insurance and provident funds) can be separated out. This would facilitate construction of a suitable financial aggregate using these assets.
\end{abstract}

Keywords: Non-parametric estimation, financial assets, India JEL Classification: E21, E41

All correspondence to:

Prof. Raghbendra Jha, Australia South Asia Research Centre, Australian National University, Canberra, Australia 0200

Phone: + 61261252683

Fax: + 61261250443

Email: r.jha@anu.edu.au

\footnotetext{
* We are grateful to Hal Varian for providing access to NONPAR routine and to Satya Narayan Murthy for help with GAMS programming. Any remaining errors are our own responsibility.
} 


\section{Introduction}

As an economy develops the structure of its savings plays a crucial role in determining the kinds of investments that will be financed by incremental savings. In a primitive, selfsufficient economy the savings are primarily in physical assets such as land. As the financial system matures the role of financial intermediation becomes critical in channelising savings into more immediately productive investments. This is particularly relevant in developing and transition economies that have been hitherto controlled and are undergoing rapid transformation through a program of structural reforms. An understanding of the structure of savings is pertinent both for increasing the volume of aggregate savings (to garner resources for higher economic growth) as well as affecting their composition (towards more productive instruments) through an understanding of inter-asset substitutability.

Central to this understanding is our understanding of the microeconomic foundations for savings behaviour. In other words, we need to understand the preference structure of households (and hence their utility functions) as they choose among various financial assets.

This paper is addressed to analysing this question in the context of Indian economy. As is well known the Indian economy has undergone rapid financial sector reform in recent years (RBI (2003)) and has, as a consequence, experienced considerable change in the composition of assets available for investment. Figure 1 depicts the enormous changes in the structure of financial savings that have taken place at the household level, particularly since the initiation of the reforms in 1991.

\section{Figure 1 here.}

A theoretical framework with ready empirical applicability devoted to understanding the behaviour of savings would be helpful, particularly in the face of changes as rapid as those evidenced in Figure 1. Two general approaches for finding a structure for the financial assets 
exist: one is the parametric and the other nonparametric. For the parametric case Longjam (2003) modelled different parametric functional forms (translog and Fourier) for financial assets in India and conducted tests for weak separability. However, this estimation was inconclusive in that it was revealed that some rejections of the demand systems could be rejections of flexible forms or the theory itself.

The logical way to break this logjam is to check whether the representative consumer's demand structure can at all be rationalized by a utility function and not assume its existence per se as the parametric approach does. A subsequent question is whether this utility function is weakly separable between some assets and its remaining arguments. If that is the case a meaningful financial aggregate using these assets can be defined since there would exist adequate degrees of freedom for statistical tests.

The nonparametric approach satisfactorily addresses these issues. It is based on the revealed preference theorem and has the advantage that, unlike the parametric form, it does not need a long data series. This is important in our case as it turns out that the number of observations (particularly post-reform) is small compared to what one would like to have for a (parametric) demand study. Another disadvantage of the parametric approach that has drawn a great deal of attention is that only a joint test of weak separability and functional forms is possible. Varian (1982) argues that this makes the functional form untestable so that it is taken essentially on faith. As Swofford and Whitney (1994) point out, this is a serious matter when it comes to testing for weak separability (and establishing the relevant financial aggregate). Barnett and Choi (1989) find the specifications of the flexible functional forms are very unreliable for the testing of weak separability. Another advantage of the nonparametric tests is that they do not require any ad hoc specification of the functional forms for demand equations and these techniques are purely dealt with finite mathematics. 
In this paper, we follow the nonparametric tests based on the revealed preference theorem, which has been developed over the years by Samuelson (1938), Houthakkar (1950), Afriat (1967) and Varian (1982) and more recently by Swofford and Whitney (1994). We implement the non-parametric tests of weakly separable preference structure of Varian (1985) and test for the necessary and sufficient conditions of the weak separability hypothesis by solving the non-linear programming equations advanced by Swofford and Whitney (1994).

The conceptualisation of the demand for financial assets is typically based on a solution to the following optimisation problem for a representative economic agent

$$
\operatorname{Max} U\left(x_{1 t}, x_{2 t}, \ldots, x_{n t}\right), i=1,2, \ldots, n
$$

subject to $\pi_{i t} x_{i t}=F_{t}$ where $x_{i t}$ is the $i^{\text {th }}$ financial asset at period $t, \pi_{i t}$ is the user cost of the $i^{\text {th }}$ asset and $F_{t}$ is the total expenditure on the financial assets. From this optimisation we could derive share demand equations under the (alternative) assumptions of the translog and Fourier functional forms for the corresponding indirect utility functions. ${ }^{1}$ This is reported in Longjam (2003) who also computes elasticities of substitution among different assets. If weak separability was satisfied, this would enable us to ascertain the preference structure of the financial assets. ${ }^{2}$ Weak sustainability will be satisfied if there exists any subutility function $V$ such that $U(x \mathbf{1}, \boldsymbol{x} \mathbf{2})=U(\boldsymbol{x} \mathbf{1}, V(x \mathbf{2}))$ where $\boldsymbol{x} \mathbf{1}=\left\{x_{1}, x_{2}, \ldots, x_{m-1}\right\}$ and $x \mathbf{2}=\left\{x_{m}, x_{m+1}, \ldots, x_{n}\right\}$ which has the same solution as maximisation problem in (1) and the elasticities of substitution among assets in $\boldsymbol{x} \mathbf{2}$ are independent of price effects on assets in $\boldsymbol{x} \mathbf{1}$. A prior question, however, is whether the available data can at all be rationalised by a utility function with desirable properties and where testing for this and weak separability can be distinct. This

\footnotetext{
${ }^{1}$ Under the assumptions that the regularity conditions are satisfied for the indirect utility function, they are also satisfied for the direct utility functions by the property of duality.

${ }^{2}$ A utility function $\mathrm{u}(\mathrm{x})$ is weakly separable in the arguments $\mathrm{x}(=(\mathrm{a}, \mathrm{b}))$ if there is a subutility function uo(b) and a macro function $u^{*}\left(\boldsymbol{a}, u_{o}(\boldsymbol{b})\right)$ which is continuous and monotonically strictly increasing in $u_{o}(\boldsymbol{b})$ and $\boldsymbol{a}$, such that $u(\boldsymbol{x})=u^{*}\left(\boldsymbol{a}, u_{o}(\boldsymbol{b})\right)$ (Swofford and Whitney (1994)).
} 
paper examines this particular issue. It examines the possibility of constructing a representative utility function that can rationalise the aggregate data.

The plan of this paper is as follows. In section II we outline the methodology adopted in the nonparametric approach. Section III presents and discusses the empirical results and section IV concludes.

\section{The Nonparametric Approach - Methodology}

We implement an algorithm developed by Varian (following Afriat (1967)) for testing revealed preference theorem. Afriat's axioms are as follows:

a) There exists a nonsatiated utility function that rationalises the data.

b) The data satisfy the axiom of transitivity, i.e., $\pi^{r} x^{r} \geq \pi^{r} x^{s}, \pi^{s} x^{s} \geq \pi^{s} x^{t}, \ldots, \pi^{q} x^{q} \geq \pi^{q} x^{r}$, implies $\pi^{r} x^{r}=\pi^{r} x^{s}, \pi^{s} x^{s}=\pi^{s} x^{t}, \ldots, \pi^{q} x^{q}=\pi^{q} x^{r}$, where $\pi^{r}=\left(\pi_{1 t}, \pi_{2 t}, \ldots \pi_{n t}\right)$ for $i=1$, $\ldots n . k$ is the number of assets and $n$ is the number of observations.

c) There exists a utility function $U^{i}$ and marginal utility $\lambda^{i} \geq 0, i=1, \ldots, n$ such that $U^{i} \leq U^{j}+\lambda^{i} \pi^{i}\left(x^{i}-x^{j}\right)$, for $i, j=1, \ldots n$

d) There exists a nonsatiated, continuous, monotonic, concave utility function that rationalizes the data

Afriat shows that if conditions b) or c) are satisfied, then the data can be rationalised by a utility function. Checking for condition c) is more burdensome as we need to solve a linear programming problem with $2 n$ variables and $n^{2}$ constraints. Varian's algorithm checks the transitivity condition, also known as Generalised Axiom of Revealed Preference (GARP).

Now revealed preference is defined as the satisfying of the following two conditions:

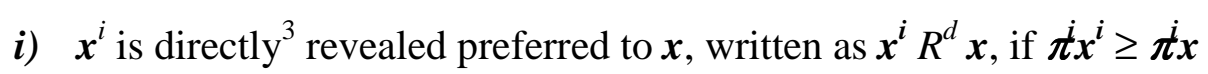

\footnotetext{
${ }^{3} x^{i}$ is strictly directly revealed preferred to $x$, written as $x^{i} R^{s} x$, if $\boldsymbol{t}^{i} x^{i}>\boldsymbol{t} \boldsymbol{x}$
} 


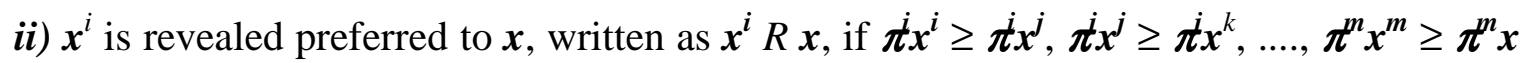
for some sequence of observations $\left(x^{i}, x^{j}, . ., x^{m}\right)$

Under this definition, then $R$ is called the transitive closure of $R^{d}$. Thus, a data set satisfies GARP if $\boldsymbol{x}^{t} R \boldsymbol{x}^{s}$ implies $\boldsymbol{\pi}^{s} \boldsymbol{x}^{s} \geq \boldsymbol{\pi}^{s} \boldsymbol{x}^{t}$ for all $t$ and $s$. Therefore, to check the consistency of the observed data with GARP (and thus with utility maximization), we need to check if there exists such a relation. Checking for the consistency of rationalising behaviour enables one to find a transitive closure. Varian suggests a way to find this transitive closure by setting up a matrix of order $T$ by $T$ where each element is either 1 if $x^{t} R^{d} x^{s}$ is true for all $t, s$ or 0 , otherwise. In most empirical work of this sort, researchers have found that writing down this matrix is actually less computationally burdensome than finding solutions to the inequality relations $U^{i} \leq U^{j}+\lambda^{i} \pi^{i}\left(x^{i}-x^{j}\right)$.

\section{Weakly separability test}

Once the data is tested for rationalisation by preference function that have the properties indicated by the revealed preference theorem, we can inquire into the weak separability of the utility function. Suppose that the utility function $U(\boldsymbol{x})$ over financial assets is weakly separable in the assets $\boldsymbol{x}=\left(x_{1}, x_{2}, \ldots x_{n}\right)$. Suppose $x_{m+1}, x_{m+2}, \ldots, x_{n}$ are separable within the $n$ assets $x_{1}, x_{2}, \ldots x_{n}$ so that the weakly separable utility maximization problem of the assets can be written as:

$$
\begin{aligned}
& \operatorname{Max} U(\mathbf{a}, \mathbf{b})=U(\mathbf{a}, V(\mathbf{b})) \\
& \text { subject to } \mathbf{p}^{\prime} \mathbf{a}+\mathbf{r}^{\prime} \mathbf{b}=Y
\end{aligned}
$$

where $\boldsymbol{p}$ and $\boldsymbol{r}$ are the respective user costs of the vector of assets $\boldsymbol{a}=\left(x_{1}, x_{2}, \ldots, x_{\boldsymbol{m}}\right)$ and $\boldsymbol{b}=$ $\left(x_{m+1}, x_{m+2}, \ldots, x_{n}\right)$ and $Y$ is total financial expenditure. Here $V(\boldsymbol{b})$ is a subutility function in $\boldsymbol{b}$ and the elasticities of the assets in the vector $\boldsymbol{b}$ are independent of any changes in the prices of the assets in the vector $\boldsymbol{a}$. 
Now, we need to ascertain whether the functions $V$ and, later $U$, hold under the conditions of Afriat's equivalent relations. The inequality relations for which we need to find the solutions are:

$$
\begin{aligned}
& U^{i} \leq U^{j}+\lambda^{j} \mathbf{p}^{j}\left(\mathbf{a}^{i}-\mathbf{a}^{j}\right)+\frac{\lambda^{j}}{\mu^{j}} r^{j}\left(V^{i}-V^{j}\right) \\
& V^{i} \leq V^{j}+\mu^{j} r^{j}\left(\mathbf{b}^{i}-\mathbf{b}^{j}\right)
\end{aligned}
$$

for positive values of $\lambda$ and $\mu$. If there is a solution to these inequality relations, then the observed data can be rationalised by a concave, monotonic, and non-satiated utility function. Further, the function is weakly separable in the variable $\boldsymbol{x} \mathbf{2}$. However, establishing this involves considerable computational burden. Varian (1985) suggests a two-stage procedure to check for weak separability. First we conduct the GARP test for the assumed groups of the assets. Once GARP is satisfied, we can consider that group as a single asset whose value is $V$ and whose price is $1 / \mu$, These magnitudes can be computed using an algorithm due to Varian (1982). ${ }^{1}$ Now taking this asset as a single commodity and clubbing the rest of the assets together, we conduct the GARP test again. If both tests are satisfied we can say that the data can be rationalised by a well-behaved weakly separable utility function.

\section{Empirical Results}

\section{Data:}

The data on the financial assets are from the RBI Handbook of Statistics on the Indian Economy 2001, CD Rom. The assets considered are a) currency, b) net deposits, c) shares and debentures, d) net claims on government, e) insurance funds, and f) provident funds. We convert (nominal values of) the assets into per capita terms by dividing the total value of each

\footnotetext{
${ }^{4}$ Varian [1982] gives a software routine where one can find $1 / \mu$.

${ }^{5}$ However, this condition is only necessary but not sufficient as Varian (1985) and Barnette and Choi (1989) point out.
} 
asset by the Indian population aged 15 and above for each year. The data set on population is collected from the IFS: IMF and spans the period from 1970 to 1998 . The real user costs and total expenditures are found out in the manner consistent with Barnett (1978) and Anderson, Jones and Nesmith (1997). Thus we have a series of 6 assets in per capita terms with their respective normalized real user costs. ${ }^{6}$ We then fit the GARP model to this data series using Varian's software routine.

\section{Testing for GARP}

We find that the data can be rationalized by a utility function and some weak separability tests are satisfied. The results are outlined in Table 1.

\section{Table 1 here.}

Table 1 gives all possible combinations of the preference structures where GARP is consistent with the given data. The $U_{i}$ denote preference structures for asset $i$. The $U$ in any column indicates that the particular asset in that row is included in the overall utility function whereas a $V$ indicates that the particular asset in the row is included in the subutility function as a result of the weak separability tests. The $Y^{\prime}$ s indicate that the preference structure satisfies the necessary condition for weak separability and is consistent with GARP. Table 1 thus basically indicates the various groupings of the assets in the subutility function and the overall utility function for which GARP is not violated. Table 1 is constructed only for those preferences, which satisfy GARP and Afriat's inequalities.

The first column of Table 1 reveals that the data are consistent with GARP and hence can be rationalized by a well-behaved utility function. Thus our nonparametric approach justifies the incorporation of financial assets in a well-behaved utility maximization framework. The second column of Table 2 reveals that contractual savings (assets 5 (LIC)

\footnotetext{
${ }^{6}$ The user costs are normalized by the total expenditure on the assets

${ }^{7}$ The explanations of the subroutines can be found from Varian [1985]
} 
and $6(\mathrm{PPF}))$, i.e., the insurance funds and provident funds ${ }^{8}$ are weakly separable from the rest of the assets. ${ }^{\natural}$ Other separability possibilities are indicated in the remaining columns.

Any violation of GARP can be due to two reasons (i) measurement error or (ii) nonoptimising behaviour of the observed data (Varian (1983)). Further, the chances of violating GARP using the non-parametric approach may vary with change in the size of the data set. Hence, it is essential to ascertain the sensitivity of the GARP test to changes in the data set. Afriat (1967) constructed an efficiency index to compute the required increase in the expenditure of good $i$ so that it is revealed preferred to good $j$. Following Afriat, we say that an observation $r$ is directly revealed preferred to an observation $s$ at efficiency level $e$ if $e p^{r}$ $x^{r} \geq p^{r} x^{s}$. $e=1$ is the standard revealed preference case and $e=0$ is vacuous. For a given $e$ we can construct the analogue of the direct revealed preference measure, compute its transitive closure, $R_{e}$ and then check the analogue of $G A R P_{e}$ : if $x^{s} R_{e} x^{t}$, then $e p^{t} x^{t} \geq p^{t} x^{s}$.

From the transitive closure we calculated in testing for the consistency of the GARP, we can calculate Afriat's index satisfying the above relations. Table 2 gives the values of Afriat's index (for 10 consecutive points) under the assumption that the utility function is $\mathrm{U}_{2}$. This reveals that the choices are $99 \%$ efficient with normalized user cost or real user cost in the preference structure $U_{2}$.

\section{Table 2 here.}

However, checking for the consistency of the GARP (and hence Afriat's efficiency index) is not sufficient for a preference structure to be weakly separable. However, checking the necessary and sufficient conditions using Varian's inequality relations is computationally

\footnotetext{
${ }^{8}$ These are sometimes known as contractual savings.

${ }^{9}$ This would imply a preference structure of the forms $U_{2}$ where $U_{2}=U(C U R, D E P, S H D B, C L A I M, V(L I C$, $P P F)$ ). However this conclusion is only tentative since this may not be the only utility function satisfying GARP and other necessary conditions. The $U$ and $\lambda$ computed from Afriat's inequality suggest that the preference structure $U_{2}$ is quite consistent over time.

${ }^{10}$ Although Afriat does not require it, we adopt the convention that it is always the case that $x^{r} R^{D} x^{r}$; i.e., an observation is always directly revealed preferred to itself.
} 
burdensome. Solutions to Varian's inequalities may not be found if the level of the total financial expenditure is not optimal and which in turn may reject the weak separability condition. Swofford and Whitney (1994) propose a generalization of the conditions for weak separability based on Varian's inequality conditions. In testing for weak separability in our data set we implement the Swofford and Whitney (1994) model since it has the advantages of allowing incomplete adjustment in allocation of expenditure among the various financial assets, e.g., if an individual faces a user-price shock (because of an immediate restriction/deregulation of interest rate of an asset), s/he may take more than a year to adjust to her/his total expenditure on the financial assets.

We use Varian's (1982) routine to find out the transitive closure. This is handled by using the Mathematica package. The results are interesting: we pass GARP test comfortably for all the six assets together. We also find that there are solutions of $U$ and $\lambda$ which satisfy the Afriat inequalities, which is another condition of checking for GARP. So whether by the transitive closure or by the Afriat's inequalities, we conclude that the observed data is consistent with GARP and hence there exist a utility function, which can rationalize the data.

We then test sufficiency conditions using the methodology of Swofford and Whitney (1994). They examine the inequality conditions and deduce the necessary and sufficient conditions for weak separability to hold, given Varian's Inequalities (3). Solutions to Varian's inequalities may not be found if the level of the total financial expenditure is not optimal since this may violate the weak separability condition. However, in the case of Swofford and Whitney's inequalities, conditions can be laid down such that any given data can be tested for weak separability.

The Swofford and Whitney (1994) methodology is equivalent to finding a solution to the following minimization problem: 


$$
\begin{aligned}
& \operatorname{Min} F=\sum\left(\tau^{j}-\mu^{j} \varphi^{j}\right)^{2} \\
& \text { sub to } \\
& U^{i} \leq U^{j}+\tau^{j} \mathbf{p}^{j}\left(\mathbf{a}^{i}-\mathbf{a}^{j}\right)+\varphi^{j}\left(V^{i}-V^{j}\right) \\
& V^{i} \leq V^{j}+\mu^{j} \mathbf{r}^{j}\left(\mathbf{b}^{i}-\mathbf{b}^{j}\right)
\end{aligned}
$$

Now we have two interpretation of the possible results to this non-linear programming problem (4):

a) if a feasible solution to this problem exists, then preferences are weakly separable in the assets in $V$

b) if a feasible solution exists and the objective function is minimized to zero, then preferences are weakly separable and adjustment is complete within each period.

The financial data described above are used for finding the solutions to the above nonlinear optimisation problem. We use a GAMS non-linear programming procedure to solve the problem. In the non-linear optimisation, with 29 observations, the number of variables comes to $5^{*} 29=145$ whereas the number of constraints they have to satisfy is very large $(2 * 29 * 28=1,624)$. Since a solution to the nonlinear programming problem (4) is a condition for the necessary and sufficient condition of weak separability test, we examine the preference structures as discussed in Table 1.

When we examined for the existence of the solutions of the various preference structures, we found many of them violating constraints or being unable to give any solutions. The only result that is consistent and is validated by a solution to (4) is the preference structure $U_{2}=$ $U(C U R, D E P, S H D B, C L A I M, V(L I C, P P F))$. Since the value of the minimand (4) goes arbitrarily close to zero, we conclude that there was complete adjustment. Since the solutions are consistent with the GARP test, we can claim that the preference structure $U 2$ satisfies the necessary and sufficient condition for weak separability and represents the preferences of a representative individual making decisions on financial assets. The fact of weak separability 
of this utility function enables one to construct a suitable financial aggregate for the Indian economy.

\section{Conclusions}

This paper has conducted a nonparametric test to examine the possibility of rationalizing the data by a utility function. The parametric test has the disadvantage that in some cases it is not possible to distinguish between a rejection of the functional form from a rejection of weak separability. In the nonparametric approach one can independently test for whether the available data on financial assets can be rationalised by a well-behaved function and then claim that there exists a structure that holds the necessary and sufficient condition for weak separability. In this paper we have used the algorithms developed by Varian (1985) for testing the revealed preference theorem. But the algorithms provided only a necessary condition. Therefore we further examine the sufficiency condition by searching for the existence of solutions to the inequality relations put forward by Swofford and Whitney [1994]. Not all preference structures, that have passed the GARP tests, have solutions to the relevant non-linear programming problem. However, one result that is borne out is that the preference structure has a sub-preference in that contractual savings (insurance and provident funds) can be separated out. This would facilitate construction of a suitable financial aggregate using these assets. 


\section{References}

Afriat, S. (1967), "The Construction of a Utility Function from Expenditure Data", International Economic Review, vol. 8, pp. 67-77.

Anderson, R., Jones, B., and J. Nesmith (1997), "Introduction to the St. Louis Monetary Services Project”, Federal Reserve Bank of St. Louis Review, vol. 79, no. 1, pp. 25-9.

Barnett, W. (1978), “The User Cost of Money”, Economics Letters, vol. 1, no. 2, pp. 145-9.

Barnett, W. and S. Choi (1989), “A Monte Carlo Study of Tests of Blockwise Weak Separability”, Journal of Business and Economic Statistics, vol. 7, no. 3, pp. 363-77.

Houthakker, H. (1950), "Revealed Preference and the Utility Function", Economica, Vol.17, no.1, pp.159-74.

Longjam, I. (2003), The Structure of Financial Savings in the Indian Household Sector, unpublished Ph.D. dissertation, IGIDR, Mumbai.

Reserve Bank of India (2003), Report on Currency and Finance, 2001-02

Samuelson, P. (1938), "A Note on the Pure Theory of Consumer Behaviour", Econometrica, vol. 5, pp. 61-71.

Swofford, J. and G. Whitney (1994), “A Revealed Preference Test for Weakly Separable Utility Maximization with Incomplete Adjustment”, Journal of Econometrics, vol. 60, nos 1-2, pp. 235-49.

Varian, H. (1982), "The Nonparametric Approach to Demand Analysis", Econometrica, vol. 50, no. 4, pp. 945-72.

Varian, H. (1983), "Nonparametric tests of Consumer Behaviour", Review of Economics and Statistics, vol. 50, no. 1, pp. 99-110.

Varian, H. (1985), "Nonparametric Analysis of Optimizing Behaviour with Measurement Error", Journal of Econometrics, vol. 30, nos.1-2, pp. 445-58.

Varian, H. (1996), Computational Economics and Finance: Modeling and Analysis with Mathematica. Santa Clara, California: Springer, The Electronic Library of Science (TELOS). 
Table 1: Results from revealed preference tests

\begin{tabular}{lccccccc}
\hline \hline Preference Structure & $U_{1}$ & $U_{2}$ & $U_{3}$ & $U_{4}$ & $U_{5}$ & $U_{6}$ & $U_{7}$ \\
\hline CUR & $U$ & $U$ & $U$ & $U$ & $U$ & $U$ & $U$ \\
DEP & $U$ & $U$ & $U$ & $U$ & $U$ & $V$ & $V$ \\
SHDB & $U$ & $U$ & $U$ & $V$ & $V$ & $V$ & $V$ \\
CLAIM & $U$ & $U$ & $V$ & $V$ & $U$ & $U$ & $V$ \\
LIC & $U$ & $V$ & $V$ & $V$ & $V$ & $V$ & $U$ \\
PPF & $U$ & $V$ & $V$ & $V$ & $V$ & $U$ & $U$ \\
\hline Test Results & & & & & & & \\
\hline GARP & $\mathrm{Y}$ & $\mathrm{Y}$ & $\mathrm{Y}$ & $\mathrm{Y}$ & $\mathrm{Y}$ & $\mathrm{Y}$ & $\mathrm{Y}$ \\
Afriat's inequalities & $\mathrm{Y}$ & $\mathrm{Y}$ & $\mathrm{Y}$ & $\mathrm{Y}$ & $\mathrm{Y}$ & $\mathrm{Y}$ & $\mathrm{Y}$ \\
Weakly Separability & $\mathrm{Y}$ & $\mathrm{Y}$ & $\mathrm{Y}$ & $\mathrm{Y}$ & $\mathrm{Y}$ & $\mathrm{Y}$ & $\mathrm{Y}$ \\
\hline
\end{tabular}

Table 2: Afriat's index number

\begin{tabular}{l}
0.7500 \\
0.8750 \\
0.9375 \\
0.9687 \\
0.9844 \\
0.9921 \\
0.9960 \\
0.9980 \\
0.9990 \\
0.9995 \\
\hline
\end{tabular}

Note: The Afriate's index e after 10 consecutive points hasn't improved beyond 0.995 


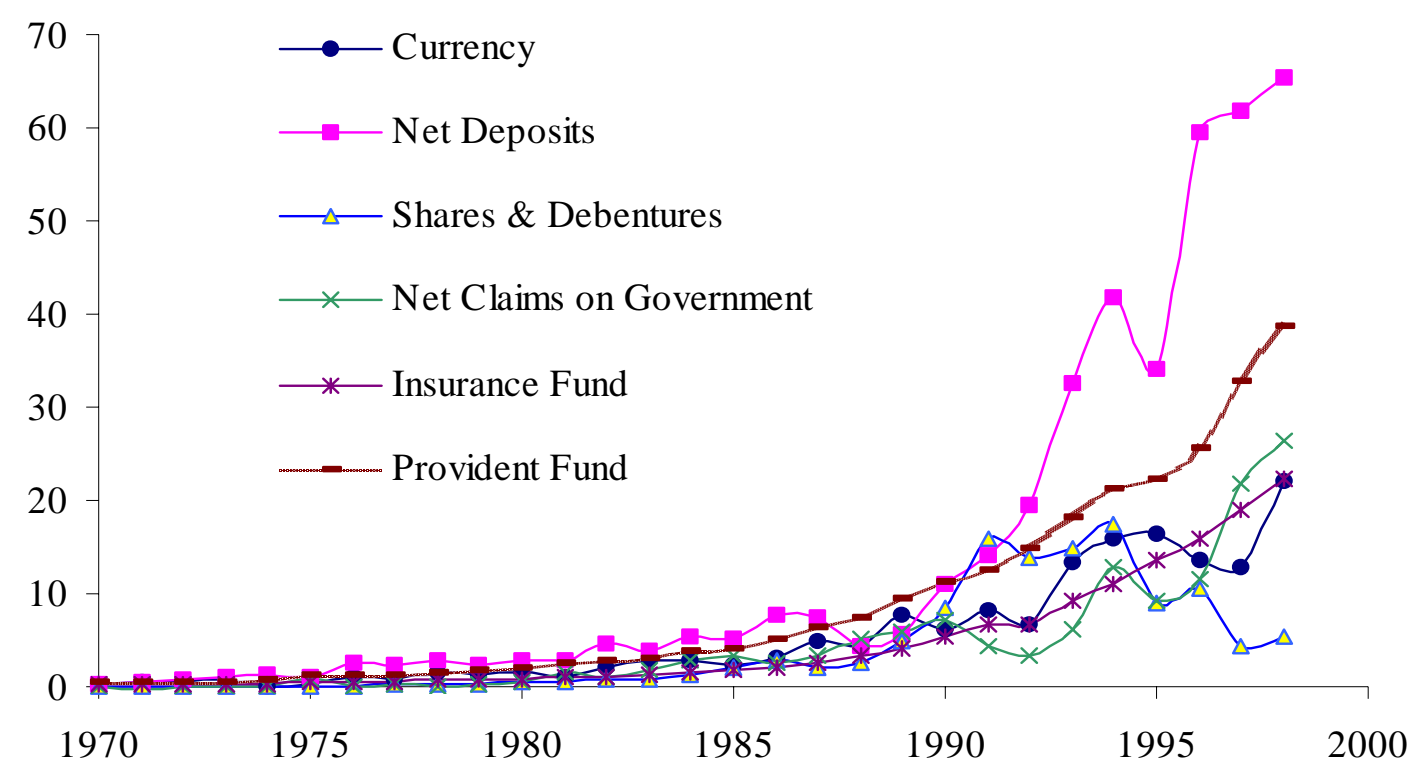

Fig. 1 Net Financial Assets in the Household Sector Savings (Rs. in 1,000 Crores) 